\title{
ReaR

\section{Prevención de la infección de herida quirúrgica. Recomendaciones del CDC 2017}

Artículo original: Berríos-Torres SI, Umscheid CA, Bratzler DW, Leas B, Stone EC, Kelz RR et al; Healthcare Infection Control Practices Advisory Committee. Centers for Disease Control and Prevention guideline for the prevention of surgical site infection, 2017. JAMA Surg. 2017;152(8):784-791. (Pubmed) (HTML)

Gordo Flores, ME, Paz Martín D.

Complejo Hospitalario de Toledo.

\section{Resumen}

Aproximadamente el $8 \%$ de los pacientes ingresados en un hospital sufren una infección asociada a la asistencia sanitaria. La infección de herida quirúrgica (IHQ), definida como la infección de la incisión, órgano o espacio tras la cirugía, supone una quinta parte del total, estimándose que aproximadamente el $5 \%$ de todos los pacientes que se intervienen de cirugía abierta desarrollarán una.

\section{Introducción}

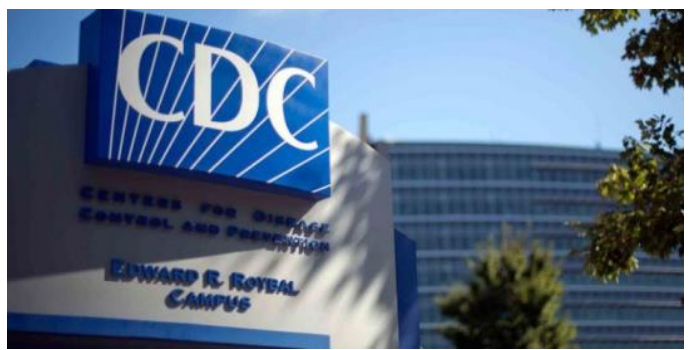

Aproximadamente el $8 \%$ de los pacientes ingresados en un hospital sufren una infección asociada a la asistencia sanitaria. La infección de herida quirúrgica (IHQ), definida como la infección de la incisión, órgano o espacio tras la cirugía, supone una quinta parte del total, estimándose que aproximadamente el $5 \%$ de todos los pacientes que se intervienen de cirugía abierta desarrollarán una.

Khuri et al (1) publicaron un estudio de 105.000 pacientes del National Surgical Quality Improvement Proyect, en el que se demostró que el desarrollo de una de las 22 complicaciones postoperatorias estudiadas, entre las que se incluían infección superficial y profunda de herida quirúrgica, reducía la esperanza de vida media en un $69 \%$ independientemente del riesgo preoperatorio del individuo.

La IHQ también afectaría a la calidad de vida del sujeto asociándose a dolor, impotencia funcional, aumento del tiempo de hospitalización o formación de lesiones cutáneas estéticamente inaceptables (2).

Resulta fundamental conocer que aproximadamente la mitad de todas las IHQ podrían evitarse mediante la aplicación de estrategias basadas en la evidencia (3).

Por todos estos motivos existe un interés creciente en cuanto a la prevención de la IHQ, que ha ido aumentando de forma paralela a la cada vez mayor complejidad perioperatoria, al incremento de número de pacientes que se intervienen y al gasto asociado a esta complicación quirúrgica. 
El Centers for Disease Control and Prevention (CDC) elabora esta guía en 2017 con el objetivo de actualizar las recomendaciones sobre prevención de la IHQ que publicó en 1999.

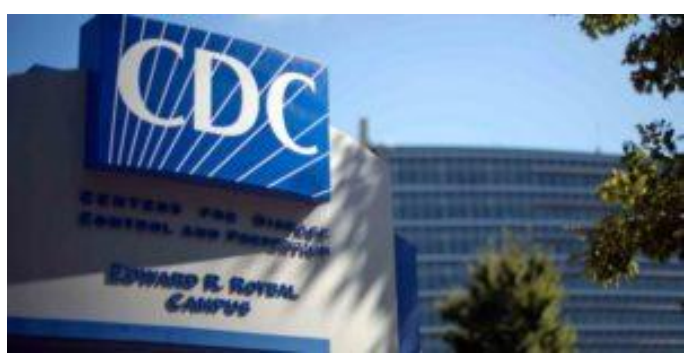

\section{Metodología}

Se realizaron revisiones sistemáticas en aspectos específicos, seleccionados por expertos clínicos, sobre la prevención de la IHQ en MEDLINE, EMBASE, CINAHL y en la Cochrane Library desde 1998 hasta Abril de 2014. Se empleó una modificación del método Grading of Recommendations, Assessment, Development, and Evaluation (GRADE) para sintetizar la calidad de la evidencia y la fortaleza de las recomendaciones.

\section{Resultados principales}

Se evaluaron de forma sistemática 5759 resúmenes y 896 textos completos por dos revisores independientes.

Las recomendaciones fueron clasificadas en diferentes categorías:

- Profilaxis con antimicrobianos parenterales.

- Profilaxis con antimicrobianos no parenterales.

- Control glucémico.

- Oxigenación.

- Profilaxis con antisépticos.

- Prótesis articular: que incluía recomendaciones en los procesos; Transfusión sanguínea; Inmunosupresores sistémicos; Corticoesteroides intraarticulares;
Anticoagulación; Traje quirúrgico; Duración de la profilaxis antimicrobiana cuando se emplea drenaje; Biofilm.

De los 42 puntos publicados, 8 fueron de Categoría IA, 4 Categoría IB, 5 Categoría II y en 25 no pudo darse una recomendación o se consideró un aspecto no resuelto.

Dentro de las 12 recomendaciones de Categoría IA o IB encontramos:

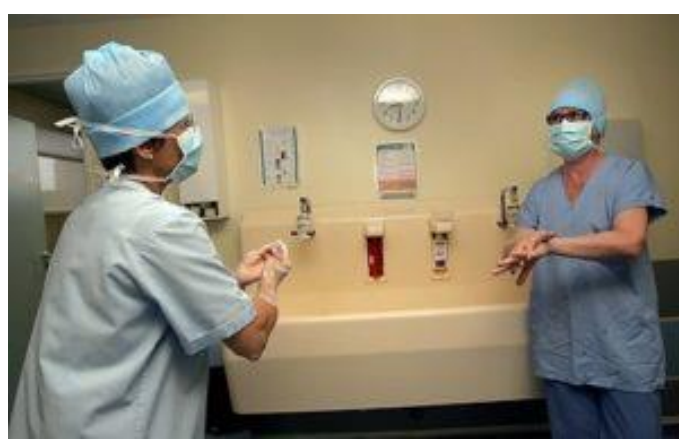

- Administrar agentes antimicrobianos

preoperatorios sólo cuando esté indicado en base a las guías de práctica clínica publicadas y con suficiente tiempo para que se haya establecido una concentración bactericida de los agentes tanto en suero como en tejidos en el momento de realizar la incisión. (Categoría IBrecomendación fuerte; práctica aceptada).

- Administrar los agentes antimicrobianos profilácticos parenterales adecuados antes de la incisión cutánea en todos los procedimientos de cesárea (no esperar al clampaje del cordón). (Categoría IArecomendación fuerte; evidencia de alta calidad).

- En procedimientos limpios y limpios-contaminados, no 
administrar dosis adicionales de agente antimicrobiano profiláctico después del cierre de la incisión quirúrgica en el quirófano, incluso en presencia de un drenaje. (Categoría IArecomendación fuerte; evidencia de alta calidad).

- La última recomendación se aplica incluso en caso de artroplastias protésicas en tratamiento con corticoesteroides sistémicos o inmunosupresores. (Categoría IArecomendación evidencia de alta calidad).

- No aplicar agentes antimicrobianos (p. ej. ungüentos, soluciones $\mathrm{o}$ polvos) a la incisión quirúrgica para la prevención de IHQ. (Categoría IBrecomendación fuerte; evidencia de baja calidad).

- Implementar control glucémico perioperatorio con el objetivo de niveles de glucosa en la sangre inferiores a $200 \mathrm{mg} / \mathrm{dL}$ en todos los pacientes (con $\mathbf{y}$ sin diabetes). (Categoría IA - recomendación fuerte; evidencia de alta a moderada calidad).

- Mantener la normotermia perioperatoria. (Categoría IA - recomendación fuerte; evidencia de alta a moderada calidad).

- Para pacientes con función pulmonar normal y sometidos a anestesia general con intubación endotraqueal, administrar FIO2 durante la cirugía y después de la extubación en el postoperatorio inmediato. $\begin{array}{llr}\text { (Categoría } & \text { IA } & - \\ \text { recomendación } & & \text { fuerte; }\end{array}$

\author{
evidencia de calidad \\ moderada).
}

- Aconseje a los pacientes ducharse o bañarse (cuerpo completo) con jabón (antimicrobiano o no antimicrobiano) o con un agente antiséptico al menos la noche anterior al día de la operación. (Categoría IBrecomendación fuerte; práctica aceptada).

- Realice la preparación intraoperatoria de la piel con un agente antiséptico a base de alcohol, a menos que esté contraindicado. (Categoría IA recomendación fuerte; evidencia de alta calidad).

- No evite la transfusión de los productos sanguíneos necesarios a los pacientes quirúrgicos como un medio para prevenir la IHQ. (Categoría IB - recomendación fuerte; práctica aceptada).

\section{Discusión}

Estas nuevas guías 2017 ofrecen una revisión sistemática de la evidencia sobre diferentes aspectos claves de la prevención de la IHQ. Sin embargo, los autores no reevaluaron muchas recomendaciones fuertes lanzadas en las guías de 1999 que hoy son consideras prácticas aceptadas.

El documento recién publicado presenta una extensión menor que el de 1999 (6 páginas frente a 38), pero incluye un suplemento de 600 páginas que detalla la extensa revisión bibliográfica que se llevó a cabo para desarrollar las recomendaciones.

Es llamativo el bajo número de recomendaciones apoyadas por pruebas claras; 8 Categorías IA de los 42 puntos tratados. Es probable, que buena parte de nuestra práctica clínica habitual en esta materia se deba más a lo que 
tradicionalmente se ha venido realizando, aunque lógico, que a la aplicación de resultados obtenidos en trabajos científicos.

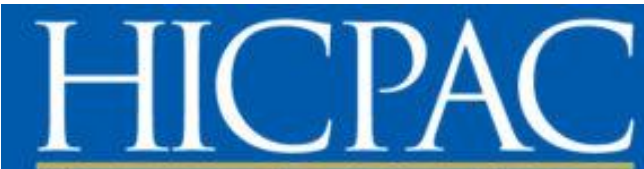

HEALTHCARE INFECTION CONTROL PRACTICES ADVISORY COMMITTEE

Desafortunadamente en muchas áreas los autores no dan recomendación o consideran que no están resueltas. Entre estas áreas no resueltas se incluyen el momento óptimo y el número de baños preoperatorios, tiempo óptimo de administración de antimicrobianos antes de la cirugía, la necesidad de dosis adicionales de antimicrobianos en cirugías largas, la inmersión de prótesis en soluciones antimicrobianas $\mathrm{O}$ antisépticas antes del implante o el nivel óptimo y la duración de la FiO2 perioperatoria. Esta última recomendación (hay varias a lo largo texto) se basa en una evidencia moderada y resulta especialmente controvertida debido a la probable ausencia de eficacia $y$ a que potencialmente puede ser lesiva. La fisiopatología perioperatoria es compleja y las poblaciones quirúrgicas son heterogéneas por lo que los autores desaconsejamos el empleo rutinario de altas concentraciones de $\mathrm{FiO} 2$ hasta que no se establezca claramente la relación riesgo/beneficio.

La impresión que podría desprenderse de la revisión de este trabajo es que no queda a la altura del actual referente mundial de la Organización Mundial de la Salud (OMS) publicado en 2016 (4), sin embargo, en este último también se aprecia, como no podría ser de otra forma, las mismas limitaciones en cuanto a la calidad de la evidencia de las recomendaciones; de las 34 expuestas 17 son de calidad baja o muy baja, 12 moderada y 5 no aplicable.

En cualquier caso, las áreas sin recomendación o no resueltas indican el futuro de próximas investigaciones con estudios bien diseñados que permitan valorar el efecto de determinadas intervenciones en la incidencia de IHQ.

\section{Bibliografía}

- Khuri SF, Henderson WG, DePalma RG, Mosca C, Healey NA, Kumbhani DJ. Determinants of long-term survival after major surgery and the adverse effect of postoperative complications. Ann Surg. 2005;242(3):326-41. (PubMed) ( $\underline{\text { HTML) }}$ (PDF)

- Evidencia en el manejo de la infección perioperatoria Paz Martín, M.B. Serna Gandía, B. Castaño Moreira En manual de infecciones perioperatorias $2^{\text {a }}$ Edición. Ed. ERGON 2017. (Libro)

- Umscheid CA, Mitchell MD, Doshi JA, Agarwal R, Williams K, Brennan PJ. Estimating the proportion of healthcare-associated infections that are reasonably preventable and the related mortality and costs. Infect Control Hosp Epidemiol. 2011;32(2):101-114. (PubMed)

- Global Guidelines for the Prevention of Surgical Site Infection. World Health Organization 2016 http://www.who.int/gpsc/ssiprevention-guidelines/en/ ( $\underline{\text { HTML}})$

Correspondencia al autor

Esther Gordo Flores esthergof@hotmail.com

MD Experta Universitaria en Seguridad del Paciente.

Servicio de Urología.

Complejo Hospitalario de Toledo.

Aceptado para blog en octubre de 2017. 